\title{
Hiperparatiroidismo primario y cáncer de paratiroides: Caso clínico*
}

\author{
Drs. LUIS ROCHA A. ${ }^{1}$, CRISTÓBAL SUAZO L. ${ }^{1}$, MIGUEL GONZÁLEZ P. ${ }^{1}$, \\ KUEN LEE CH. ${ }^{1}$, GONZALO ROSSEL D. ${ }^{1}$. \\ 1 Equipo Cirugía Cabeza y Cuello \& Plástica Maxilofacial, Servicio de Cirugía, Complejo Asistencial Barros \\ Luco Trudeau. Santiago, Chile.
}

\begin{abstract}
Primary hyperparathyroidism and parathyroid cancer. Report of one case

We report a 51 years old female operated for a primary hyperparathyroidism that relapsed in two occasions. After the first and second operation, the pathological study of the excised glands disclosed an adenoma. After the second relapse, a parathyroid gland in the right lateral and upper cervical region, located with technetium (Tc)-99m Sestamibi scintigraphy, was excised. The pathological study of the surgical piece disclosed a parathyroid carcinoma without local lymph node involvement. A postoperative $18 \mathrm{~F}$-fluorodexyglucose positron emission tomography did not show tumor dissemination.
\end{abstract}

Key words: Hyperparathyroidism, parathyroid cancer, parathyroid adenoma.

\section{Resumen}

Presentamos un caso de sexo femenino, operada por hiperparatiroidismo primario, con 2 recidivas posteriores. En las 2 primeras oportunidades, el diagnóstico histológico fue compatible con adenoma. La última intervención se debió realizar por una glándula ubicada, de acuerdo al Sestamibi Tc-99m, en la región cervical lateral alta derecha. Se realizó exploración supraomohiodea, efectuando la extirpación de la glándula y adenopatías peritumorales. La biopsia definitiva informó carcinoma de paratiroides y ganglios linfáticos negativos para neoplasia. Se realizó estudio de diseminación y PET- FDG de control en el postoperatorio alejado, sin nuevos hallazgos. Entre la primera y la segunda intervención, se realizó una tiroidectomía total por un bocio uninodular, cuya biopsia informó 3 paratiroides normotípicas, intratiroideas. En suma, se lograron identificar histológicamente 6 glándulas paratiroides.

Palabras clave: Cáncer, paratiroides, hiperparatiroidismo.

\section{Introducción}

El carcinoma de paratiroides es una neoplasia maligna endocrina poco común, descrita por primera vez por De Quevain, en 1904'. El comportamiento de esta entidad se ha descrito como de evolución lenta, pero progresiva. Tiene tendencia a infiltrar localmente, a diseminarse a linfonodos regionales y a presentar, eventualmente, metástasis pulmonares. Menos frecuentemente, se describen también

*Recibido el 12 de Marzo de 2010 y aceptado para publicación el 11 de Abril de 2010.

Correspondencia: Dr. Luis Rocha A.

Hernando de Magallanes 1042, Dpto. 1404, Las Condes Santiago, Chile.

E-mail: luisferocha@yahoo.es 
localizaciones secundarias hepáticas y óseas ${ }^{2-4}$. La mayoría de los tumores son funcionantes, secretando altos niveles de paratohormona, desencadenando calcemias bastante elevadas. La mortalidad se asocia a enfermedad regional, metástasis a distancia y a complicaciones secundarias a la hipercalcemia ${ }^{5}$. En el presente trabajo, se reporta el caso de una paciente operada por hiperparatiroidismo primario, con 2 recidivas, cuyo último informe histológico evidenció un carcinoma de paratiroides. Se efectúa, además, una revisión de la literatura.

\section{Caso clínico}

Presentamos el caso de una paciente de sexo femenino, de 51 años al momento de su ingreso al Hospital Barros Lucos Trudeau, en julio de 1988. Es hipertensa en tratamiento, único antecedente mórbido. Su motivo de derivación, fue dolor óseo generalizado, asociado a una calcemia de $15,9 \mathrm{mg} / \mathrm{dl}$.

Se realizó evaluación clínica y de laboratorio, destacando fosfatasas alcalinas de $868 \mathrm{U} / \mathrm{L}$. Una ecografía abdominal evidencia litiasis renal bilateral y un cintigrama óseo, se informa como sugerente de hiperparatiroidismo. Se solicita paratohormona (PTH) y cintigrama paratiroideo. La PTH (RIA) se informa con un valor mayor de $12 \mathrm{ng} / \mathrm{mL}$ (normal: menor de 1,6 ng/mL). Cintigrama sin hallazgos patológicos. Se realiza extirpación de paratiroides inferior derecha, informando la biopsia: fragmento de $3 \mathrm{~cm}$, de color pardo amarillento, compatible con adenoma paratiroideo.

La paciente permanece en control en endocrinología, cursando con calcemias y paratohormona dentro de rangos normales, exceptuando una oportunidad, en la que se pesquisa una calcemia de $10,8 \mathrm{mg} /$ dl. Un cintigrama paratiroideo de control, resulta normal. En las calcemias posteriores, el valor volvió a su normalidad y la paciente se mantuvo en observación.

El año 2000, por medio de una ecografía de partes blandas de cuello, se pesquisa un bocio uninodular derecho. Se realiza una punción (PAAF), la que informa neoplasia folicular, por lo que se realiza tiroidectomía total, sin incidentes. La paciente continúa controles en el policlínico de la especialidad. La biopsia diferida informa tiroiditis linfocítica crónica inespecífica, 1 paratiroides normotípica en el lóbulo izquierdo y 2 paratiroides normotípicas en lóbulo derecho, todas intratiroideas. Su calcemia de control se informa en $13 \mathrm{mg} / \mathrm{dL}$.

Se efectúa nuevo cintigrama, que revela tejido paratiroideo hiperfuncionante, en relación al polo superior del lóbulo tiroideo derecho. Su PTH intacta es de $756 \mathrm{pg} / \mathrm{ml}$. Se realiza extirpación de paratiroi- des superior derecha. La biopsia informa formación nodular de 2,5 cm, consistencia firme, café rojiza, compatible con adenoma paratiroideo. En controles postoperatorios se comprueban PTH y calcemias normales.

La paciente se mantiene asintomática hasta diciembre del año 2006, ocasión en la que presenta síntomas de hiperparatiroidismo. Se solicitan exámenes que informan PTH de $377 \mathrm{ng} / \mathrm{mL}$, calcemia de $12,7 \mathrm{mg} / \mathrm{dL}$ y Sestamibi Tc-99m que revela hipercaptación bajo la glándula submandibular derecha (Figura 1). En enero de 2007, se realiza extirpación del tumor y ganglios de grupos IIA y III ipsilaterales.

La biopsia definitiva informó neoformación compuesta por células de tipo paratiroideas, con núcleos redondos u ovalados, anisocariosis con actividad mitótica de más de 20 por 10 campos de aumento mayor, sábanas neoplásicas divididas por gruesas bandas fibrosas hialinizadas, invasión capsular y vascular, compatible con carcinoma paratiroideo; grupo IIA, 5 ganglios y grupo III, 6 ganglios, todos negativos para cáncer. No se realizó inmunohistoquímica, pues no se disponía de ella.

En el control ambulatorio, se completó estudio de diseminación y PET-FDG, los que no evidenciaron nuevos hallazgos. Sus calcemias de control al igual que la $\mathrm{PTH}$, hasta el $5^{\circ}$ mes postoperatorio, resultaron normales. Luego de ello, la paciente se mantiene en controles, fuera del hospital. Fue presentada en Comité Oncológico, donde se decidió no realizar Radioterapia, por considerarse extirpado el tumor en su totalidad, con márgenes quirúrgicos libres.

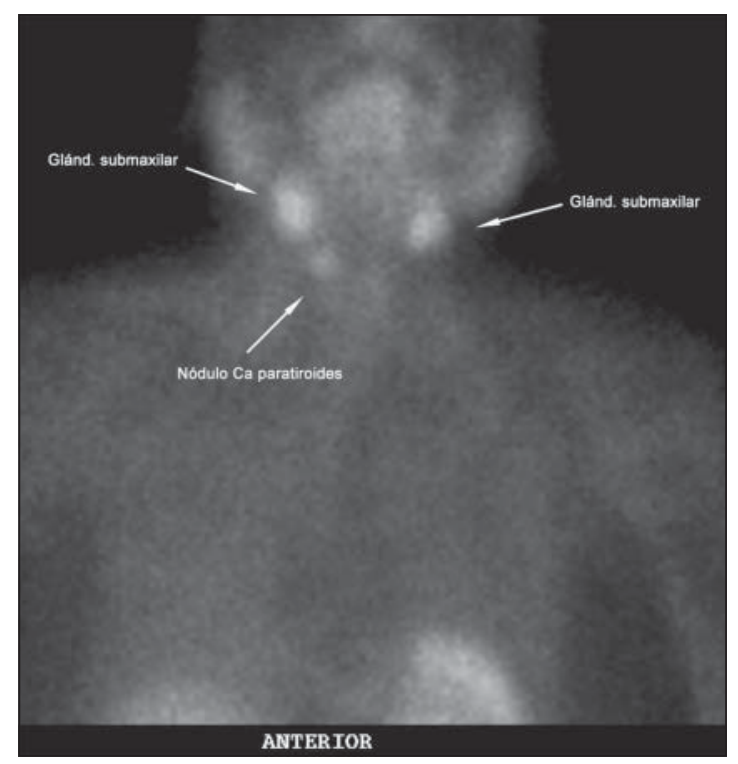

Figura 1. Cintigrama Paratiroideo Sestamibi Tc-99m. 


\section{Discusión}

La incidencia del carcinoma de paratiroides se describe como menos del $1 \%$ de los casos de hiperparatiroidismo primario ${ }^{6,7}$. Las publicaciones describen igual distribución según sexo ${ }^{6,8}$. El rango de edad promedio de presentación, se encuentra entre los 40 y 55 años.

Se presenta como una entidad aislada o puede asociarse a un síndrome como NEM tipo I, IIA o hipercalcemia hipocalciúrica familiar ${ }^{9-11}$. La clínica y el laboratorio pueden sugerir el diagnóstico, pero los hallazgos son inespecíficos. Calcemias sobre 14 $\mathrm{mg} / \mathrm{dL}$ son muy sugerentes. La PTH está elevada habitualmente entre 3 a 10 veces el valor normal ${ }^{12-14}$. Los órganos más frecuentemente afectados por la hipercalcemia, son el riñón y el hueso ${ }^{2,6,12}$. Además de ello, estos pacientes tienen un elevado riesgo de presentar pancreatitis aguda, enfermedad ulcerosa y anemia. La morbimortalidad se asocia a enfermedad regional, metástasis a distancia, pero principalmente, a la severidad de la hipercalcemia.

Sólo un $50 \%$ de los tumores presentan morfología sospechosa en el intraoperatorio y alrededor de un $15 \%$ compromete las estructuras contiguas, incluyendo tiroides, musculatura pretiroidea y nervio laríngeo recurrente $2,3,15,16$.

Shantz y Castleman ${ }^{17}$, establecieron una serie de criterios histopatológicos para el diagnóstico en 1973:

1. Presencia de una cápsula fibrosa.

2. Arquitectura celular de tipo trabecular.

3. Presencia de figuras mitóticas.

4. Presencia de invasión capsular o vascular.

Stojadinovic ${ }^{18}$ describió las características morfológicas de este cáncer:

1. Patrón de crecimiento trabecular.

2. Bandas fibrosas densas.

3. Invasión al músculo esquelético adyacente.

4. Invasión capsular.

5. Invasión vascular.

Con el análisis de Inmunohistoquímica se han detectado algunas proteínas que se presentan con mayor frecuencia en el carcinoma que en el adenoma. Ki-27 se reporta en un $27 \%$ de los pacientes con carcinoma, y un $2 \%$ de los pacientes con adenoma. La proteína del Retinoblastoma, ha sido pesquisada con mucha mayor frecuencia en los pacientes con adenoma que en aquellos con carcinoma, en los que incluso puede estar ausente; sin embargo, ninguno de estos exámenes constituye criterio diagnóstico, por ser muy inespecíficos ${ }^{18,19}$.

El estudio de medicina nuclear, Sestamibi Tc$99 \mathrm{~m}$, es efectivo en la localización de glándulas pa- ratiroides hiperactivas $\mathrm{y}$, si se asocia a una ecografía de partes blandas de cuello, aumenta la sensibilidad para su ubicación.

En cuanto al tratamiento, muchos estudios han puesto énfasis en la resección en bloque del lóbulo tiroideo, el istmo y los ganglios del grupo central. Cuando este procedimiento es efectuado como acto terapéutico inicial, constituye la mejor opción curativa para el paciente $3,15,16$.

La radioterapia ha sido citada en la literatura, sin embargo, los datos son escasos y sus resultados discutibles. Su utilidad estaría dada en aquellos casos con márgenes positivos de resección o para disminuir la recurrencia local. Existen muy pocos antecedentes para considerarla como opción de tratamiento único ${ }^{20}$.

En cuanto a la quimioterapia, se reporta en algunas publicaciones, su utilización para el manejo de hipercalcemias severas, ultilizando 5-Fluoracilo o Ciclofosfamida, en caso de enfermedad metastásica a distancia.

La recidiva posterior a la excisión quirúrgica es común con rangos entre un 38 y $78 \%$, entre 1 mes y 20 años, con un promedio entre 2 y 5 años. La sobrevida estimada a 5 y 10 años es 85 y $49 \%$ respectivamente $^{12}$.

El caso expuesto previamente, se desarrolla en un intervalo de 18 años, donde la paciente cursó largos períodos asintomática, observándose nuevos hallazgos patológicos en dos oportunidades.

En relación a este comportamiento, parece interesante hacer referencia al Síndrome de Hiperparatiroidismo-Tumor Maxilar, enfermedad hereditaria autosómica dominante, relacionada al cromosoma 1. Esta origina adenomas paratiroideos, fibromas osificantes de los maxilares, además de diversos tipos de tumores renales. En relación al Hiperparatiroidismo, se describe el desarrollo de otros adenomas tras resecar el primero, hasta varios años después. Se pesquisan nuevamente calcemias elevadas, habiendo tenido valores normales, luego de la resección del primer adenoma. Se informa una incidencia de carcinoma hasta en un $5 \%$, en estos pacientes.

Hasta el día de hoy, nuestro hospital no cuenta con Gamma Probe ni PTH intraoperatoria, elementos que podrían haber sido relevantes, eventualmente, en disminuir el número de cirugías de este paciente. A su vez, el contar con una ecografía tiroidea, en el estudio de un paciente con Hiperparatiroidismo, puede evidenciar un bocio que requiera resolución quirúrgica, lo que permitiría evitar una reintervención.

Destacamos el número de glándulas paratiroides encontradas, luego de cuatro intervenciones, llegando a un total de seis, de las cuales tres resultaron intratiroideas. En la literatura, se describe alrededor 
de un $13 \%$ de los adultos con más de 4 glándulas y un 3\% que tendría sólo 3. La última paratiroides extirpada se ubicó en un lugar atípico, la zona cervical alta; hecho que se presentaría hasta en un $2 \%$ de los casos, en glándulas ectópicas.

Al conocerse el diagnóstico histológico de carcinoma paratiroideo, no se contaba con inmunohistoquímica. Sin embargo, tampoco se efectuó en forma diferida, por no ser un elemento definitorio en el diagnóstico. Es importante recalcar que la sospecha de éste, se genera por los criterios clínicos, de laboratorio, intraoperatorios e histológicos. Sin embargo, la certeza va a estar dada, finalmente, por la recurrencia local, las metástasis regionales o a distancia.

Considerando que la paciente permaneció con calcemias normales luego de extirpada la última paratiroides, la sospecha de que exista enfermedad no diagnosticada debe plantearse, así como también, la eventual persistencia in situ de fragmentos de aquellas paratiroides resecadas, descritas como intratiroideas.

Un seguimiento periódico es indispensable, pues las recurrencias pueden ser bastante tardías, como se ha mencionado anteriormente.

\section{Referencias}

1. De Quevain F. Parastruma maligna aberrata. Deutsche Zeitschr Chir 1904; 100: 334-352.

2. Holmes E, Morton D, Ketcham A. Parathyroid carcinoma: a collective review. Ann Surg 1969; 169: 631-640.

3. Fujimoto Y, Obara T. How to recognize and treat parathyroid carcinoma. Surg Clin N Am 1987; 67: 343-357.

4. Shane E. Parathyroid carcinoma. J Clin Endocrinol Metab 2001; 86: 485-493.

5. Giesler G, Beech D. Nonfunctional parathyroid carcinoma. J Natl Med Assoc 2001; 93: 251-255.

6. Shane E, Bilezikian J. Parathyroid carcinoma: a review of 62 patients. Endocr Rev 1982; 3: 218-226.

7. Sandelin K, Auer G, Bondeson L, Grimelius L, Farnebo L. Prognostic factors in parathyroid cancer: a review of 95 cases. World J Surg 1992; 16: 724-731.

8. Hundahl S, Fleming I, Fremgen A, Menck H. Two hun- dred eighty-six cases of parathyroid carcinoma treated in the US between 1985-1995, a National Cancer Data Base report. Cancer 1999; 86: 538-544.

9. Jenkins P, Satta M, Simmgen M, Drake W, Williamson $\mathrm{C}$, Lowe D, et al. Metastatic parathyroid carcinoma in the MEN2A syndrome. Clin Endocr 1997; 41: 747-751.

10. Hamill J, Maoate K, Beasley S, Corbett R, Evans J. Familial parathyroid carcinoma in a child. J Paediatr Child Health 2002; 38: 314-317.

11. Dionisi S, Minisola S, Pepe J, De Geronima S, Paglia F, Memeo L, et al. Concurrent parathyroid adenomas and carcinoma in the setting of multiple endocrine neoplasia type 1: presentation as hypercalcemic crisis. Mayo Clin Proc 2002; 77: 866-869.

12. Wynne A, Heerden J, Carney A, Fitzpatric L. Parathyroid carcinoma: clinical and pathologic features in 43 patients. Medicine 1992; 71: 197-205.

13. Cordeiro A, Montenegro F, Kulcsar M, Dellanegra L, Tavares M, Michaluart P, et al. Parathyroid carcinoma. Am J Surg 1998; 175: 52-55.

14. Chen Q, Kaji H, Nomura R, Sowa H, Yamauchi M, Tsukamoto $\mathrm{T}$, et al. Trial to predict malignancy of affected parathyroid glands in primary hyperparathyroidism. Endocr J 2003; 50: 527-534.

15. Snell S, Gaar E, Stevens S, Flynn M. Parathyroid cancer, a continued diagnostic and terapeutic dilemma: report of four cases and review of the literature. Am Surg 2003; 69: 711-718.

16. Shane E. Parathyroid carcinoma. J Clin Endocrinol Metab 2001; 86: 485-493.

17. Schantz A, Castleman B. Parathyroid carcinoma: a study of 70 cases. Cancer 1973; 31: 600-605.

18. Stojadinovic A, Hoos A, Nissan A, Dudas M, CordonCardo C, Shaha A, et al. Parathyroid neoplasms: clinical, histopathological, and tissue microarray-based molecular analysis. Hum Pathol 2003; 34: 54-64.

19. Cetani F, Pardi E, Viacava P, DiPollinaG, Fanelli G, Picone A, et al. Areappraisal of theRb1 gene abnormalities in the diagnosis of parathyroid cancer. Clin Endocrinol 2004; 60: 99.

20. Munson N, Foote R, Northcutt R, Tiegs R, Fitzpatrick L, Grant C, et al. Parathyroid carcinoma: is there a role for adjuvant radiation therapy? Cancer 2003; 98: 23782384. 